\title{
Intoxicação parenteral por herbicida: estamos preparados para o cuidar?
}

RESUMO | Objetivo: relatar o caso de uma paciente jovem admitida na Unidade de Terapia Intensiva (UTI), vítima de suicídio por envenenamento parenteral por paraquat (PQ) e propor os principais diagnósticos de enfermagem e intervenções de acordo com a Classificação Internacional para as Práticas de Enfermagem(CIPE®). Método: Estudo descritivo retrospectivo do tipo estudo de caso, de uma paciente admitida na unidade de terapia intensiva pública no interior sul da Amazônia legal. Relato de caso: a vítima injetou PQ no bíceps, o local evoluiu para induração e necrose por coagulação, conforme avanço e metabolização do $\mathrm{PQ}$ a paciente apresentou manifestações, como leucocitose, lesão hepatorenal, respiratória e em seguida falência múltiplas de órgãos (FMO). Conclusão: Conclui-se que vítimas de intoxicação por PQ, são considerados vítimas de tentativa de suicídio, a ingestão oral acima de 40/45mg/kg aumenta significativamente a mortalidade para 100\%, e a administração parenteral contribui diretamente com FMO, e óbito em 100\%.

Palavras-chaves: Paraquat; Herbicida; Intoxicação; Enfermagem; Unidade de terapia intensiva.

\begin{abstract}
Objective: report the case of a young patient admitted to the Intensive Care Unit (ICU), victim of suicide due to paraquat $(\mathrm{PQ})$ parenteral poisoning and propose the main nursing diagnoses and interventions according to the International Classification for Nursing Practices (ICNP®). Method: retrospective descriptive study of the case study type, of a patient admitted to the public intensive care unit in the southern interior of the legal Amazon. Case report: the victim injected PQ into the biceps, the site evolved to induration and necrosis by coagulation, as the PQ progressed and metabolized, the patient presented with manifestations such as leukocytosis, hepatic and respiratory damage and then multiple organ failure (FMO). Conclusion: It is concluded that victims of PQ intoxication are considered victims of attempted suicide, oral intake above $40 / 45 \mathrm{mg} / \mathrm{kg}$ significantly increases mortality to $100 \%$, and parenteral administration contributes directly to $\mathrm{FMO}$, and death in $100 \%$.
\end{abstract}

Keywords: Paraquat; Herbicide; Intoxication; Nursing; Intensive care unit.

RESUMEN | Objetivo: informar el caso de un paciente joven ingresado en la Unidad de Cuidados Intensivos (UCI), víctima de suicidio debido a intoxicación parenteral por Paraquat (PQ) y proponer los principales diagnósticos e intervenciones de enfermería de acuerdo con la Clasificación Internacional de Prácticas de Enfermería(CIPE $®)$. Método: estudio descriptivo retrospectivo del tipo de estudio de caso, de un paciente ingresado en la unidad pública de cuidados intensivos en el interior del sur de la Amazonía legal. Informe del caso: la víctima inyectó PQ en el bíceps, el sitio evolucionó a induración y necrosis por coagulación, a medida que la PQ progresaba y se metabolizaba, el paciente presentaba manifestaciones tales como leucocitosis, daño hepático y respiratorio y luego falla orgánica múltiple (FMO). Conclusión: se concluye que las víctimas de intoxicación PQ se consideran víctimas de intento de suicidio, la ingesta oral por encima de $40 / 45 \mathrm{mg} / \mathrm{kg}$ aumenta significativamente la mortalidad al 100\%, y la administración parenteral contribuye directamente a la FMO, y la muerte en $100 \%$.

Palavras claves: Paraquat; Herbicida; Intoxicación; Enfermería; Unidad de terapia intensiva.

\section{Laurindo Pereira de Souza}

Doutorando em Ciências da Saúde pelo Instituto de Assistência Médica ao Servidor Público do Estado de São Paulo/AMSPE/SP, Mestre em Ciências da Saúde-IAMSPE/SP, Especialista Enfermagem em Terapia Intensiva pela Associação Brasileira de Enfermagem em Terapia Intensiva-ABENTVAMIB, Membro do departamento de enfermagem da AMIB (20202021). Coordenador do Programa de Residência Multiprofissional em UTI Cacoal/Rondônia (Brasil). ORCID: 0000-0002-9890-2621

\section{Marcia Guerino de Lima \\ Enfermeira especialista em Obstetrícia e Obstetrícia Social, docente do departamento de enfermagem da Faculdade de Ciências Biomédicas de Cacoal-FACIMED/Rondônia (Brasil). Enfermeira Obstetra do Hospital Municipal da Secretaria de Saúde de Ji- Paraná Rondônia (Brasil). \\ ORCID: 0000-0002-8303-1480}

\section{Renata Andrea Pietro Pereira Viana}

Diretora do Serviço de Terapia Intensiva do Hospital do Servidor Público Estadual de São Paulo. Doutorado em Ciências da Saúde pela UNIFESP. Presidente do Departamento de Enfermagem da AMIB (2020-2021). ORCID:0000-0001-7539-3003

\section{Rafael Ayres Romanholo}

Doutor em Desenvolvimento Regional e Meio Ambiente pela Universidade Federal de Rondônia (UNIR). Professor do Instituto Federal de Rondônia - IFRO Campos de Cacoal/RO - Pesquisador do Grupo de Pesquisa em Motricidade Humana Saúde e Sociedade (IFRO).

ORCID: 0000-0002-3210-3448

Recebido em: 15/07/2020

Aprovado em: 16/07/2020

\section{Cídia Vasconcellos}

Pós Doutorado pela USP, Orientadora do programa de Pós graduação stricto sensu em Ciências da Saúde IAMSPE/SP(Brasil) e da Faculdade de Medicina da Universidade de São Paulo-FMUSP.

ORCID: 0000-0003-3055-1084

\footnotetext{
*Artigo extraído da tese de Doutoramento em Ciências da Saúde "Intoxicações exógenas em humanos: um estudo sobre o levantamento clínico-epidemiológico dos atendimentos em Cacoal-Rondônia/ Brasil", vinculado ao programa de pós-graduação do Instituto de Assistência Médica ao Servidor Público Estadual do Estado de São Paulo-IAMSPE/SP.
} 
INTRODUÇÃO

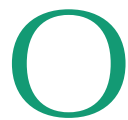
Paraquat (PQ) é hoje um dos herbicidas mais utilizados no Brasil, extremamente tóxico, quanto ocorre absorção dérmica, digestiva ou respiratória, sendo sua toxicidade principalmente observada nos pulmões. ${ }^{(1,2)}$. Em países como a Suécia, o PQ é proibido desde 1983 por causa de sua ação tóxica irreversível e pelo grande risco de acidentes fatais.

Segundo Melchiorri et al. ${ }^{(3)}$, este herbicida é capaz de produzir intoxicações fatais em humanos, promove a síndrome de transtorno respiratório e dependendo de como foi o contato, pode causar morte em menos de 30 dias. Com índice de mortalidade superior a $70 \%$, o maior risco de envenenamento ocorre durante a diluição, porém as mortes acidentais e o suicídio por ingestão são relativamente comuns. ${ }^{(4-6)}$

Segundo Pinheiro ${ }^{(7)}$ e Crugeiras et al, ${ }^{(4)}$ a intoxicação por PQ tem, um predomínio alto de complicação na função pulmonar, hepato-celular, renal e do sistema nervoso central, e isso justifica a evolução para falência de múltiplos órgãos, com taxa de letalidade em média de $100 \%$ quando ingeridos mais que 40/45mg/kg.

A Organização Mundial de Saúde $(\mathrm{OMS})^{(8)}$, relata que o suicídio é uma das dez maiores causas de mortalidade em todos os países, e uma das três maiores causas de morte na faixa etária de 15 a 35 anos em países desenvolvidos, considerando assim um problema universal de saúde pública, em que $90 \%$ dos suicídios o indivíduo tinha ou tem um problema mental, reforça ainda que em 2012 ocorreram 804.000 suicídios em todo o mundo.

Para o Ministério da Saúde ${ }^{(9)}$, do total de 8.697 casos de intoxicação atribuídos às tentativas de suicídio, $11,6 \%$ foram provenientes do uso de agrotóxicos. Além disso, em 2001, registrou 5.384 casos de intoxicação provocados por agrotóxicos no país, correspondendo a 7,1\% do total das intoxicações. Dados de 2019(10), revelaram que as intoxicações exógenas estão entre a se-
Para o Ministério da Saúde ${ }^{(9)}$, do total de 8.697 casos de intoxicação atribuídos às tentativas de suicídio, $11,6 \%$ foram provenientes do uso de agrotóxicos. Além disso, em 2001, registrou 5.384 casos de intoxicação provocados por agrotóxicos no país, correspondendo a $7,1 \%$ do total das intoxicações. gunda causa de suicídio no Brasil, com um total de 19.483 vítimas com idade superior a 20 anos em ambos os sexos.

Cabe ressaltar que a prevalência de manifestações clínicas depressivas e suicidas, corresponde ao processo e causas de autoextermínio pela própria vítima, entre os profissionais da saúde é extremamente elevada, um dos fatores de maior destaque são as condições e sobre carga de trabalho exposta a esses profissionais (11). Assim, a unidade de terapia intensiva-UTI, é um setor que presta assistência a doentes graves e de risco, e que precisam ser cuidados por profissionais de enfermagem 24horas por dia de forma ininterrupta, para tanto esses profissionais precisam estar bem consigo mesmo para prestarem uma assistência de enfermagem humana, segura e de qualidade.

Ademais, é necessário estar dotado de saberes, com capacidade, habilidades e atitudes para garantir rigor técnico e científico na execução dos cuidados de enfermagem, buscando sempre educação continuada com o intuito de melhorar e intensificar ainda mais suas práticas clínicas na garantia de um cuidado seguro e humanizado.

Por conseguinte, esta pesquisa tem por objetivo relatar o caso de uma paciente jovem admitida na Unidade de Terapia Intensiva (UTI), vítima de suicídio por envenenamento parenteral por PQ e propor os principais diagnósticos de enfermagem e intervenções de acordo com a CIPE $囚$.

\section{MÉTODO}

Trata-se de um relato de caso do tipo descritivo retrospectivo, de uma paciente do gênero feminino com 24 anos de idade admitida na UTI do Hospital Regional de Cacoal-HRC-Rondônia/Brasil, após tentativa de autoextermínio por contato com o PQ.

O recorte temporal de busca das informações foi de 2012 a junho 2018, a coleta de dados ocorreu no mês de maio a junho de 2018 e organizou-se em duas fases, sendo a primeira, o acesso as in- 
formações através do banco de dados do Sistema de Informações sobre Agravos de Notificação (SINAN) para organização dos resultados da tese de Doutorado do Programa de Pós-graduação em Ciências da Saúde-IAMSPE/SP "Intoxicações exógenas em humanos: um estudo sobre o levantamento clínico-epidemiológico dos atendimentos em Cacoal-Rondônia/ Brasil" e observou presença de um caso inédito registrado na plataforma SINAN concernente a região de Rondônia, em que a vítima tinha injetado $P Q$ pela via parenteral no bíceps.

A segunda fase, foi através do acesso ao prontuário da vítima no arquivo de prontuários do HRC, os dados clínicos pertinentes ao caso relatado foram registrados em um instrumento construído pelos próprios pesquisadores, extraiu todas informações clínicas registradas pelos profissionais da saúde no prontuário e que pudessem responder ao objetivo proposto, as informações clínicas e exames laboratoriais ilegíveis foram excluídas, e após os dados foram agrupados em

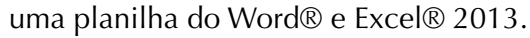

O agrupamento e raciocínio clínico diagnóstico foram feitos para identificar os diagnósticos de enfermagem fundamentados por três etapas: histórico e/ou coleta de informações, análise, interpretações e a denominação dos diagnósticos de enfermagem concernentes a vítima de intoxicação

\section{Figura 01: Mapa do Brasil, Rondônia e o local onde ocorreu a intoxicação parenteral por PQ, 2018.}

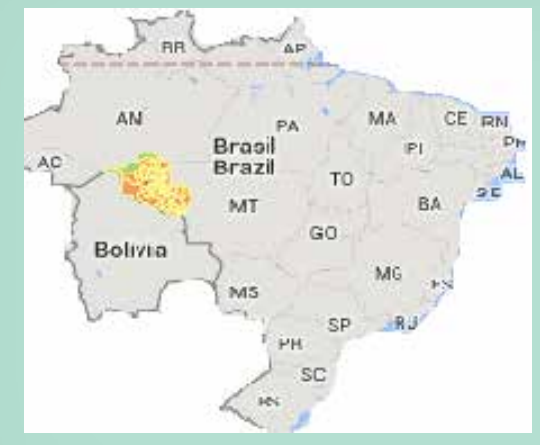

Brasil/Rondônia

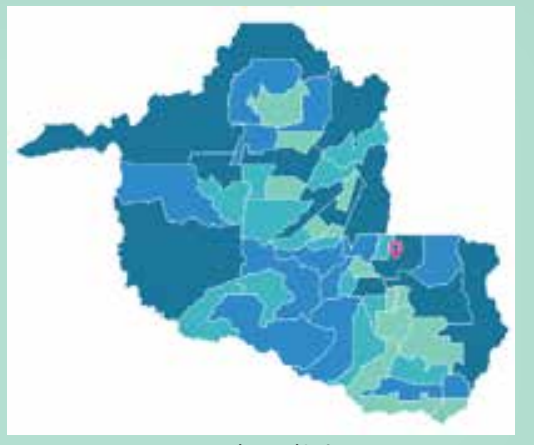

Cacoal/Rondônia
Fonte: IBGE, 2018. ${ }^{(14)}$ parenteral por PQ admitido na UTI. ${ }^{(12)}$

Para a sistematização do caso clínico e assim organizar as informações para o raciocínio clínico utilizou-se a Classificação Internacional para a Prática de Enfermagem (CIPE®), uma vez que a mesma é considerada uma terminologia ampla e complexa que representa o domínio da prática da Enfermagem, é uma norma internacional para facilitar a coleta, armazenamento e análise de dados de enfermagem por meio de raciocínio e definições de saúde. ${ }^{(13)}$

As informações foram obtidas após a anuência do Comitê de ética e pesquisa-CEP da Faculdade de Ciências Biomédicas de Cacoal (FACIMED), via Plataforma Brasil, com o número CAAE: 70461317.7.0000.5298, parecer $\mathrm{n}^{\mathrm{o}} 2.181 .872$ e parecer de emenda $\mathrm{n}^{\circ}$ : 2.506.518.

\section{RESULTADOS}

\section{Relato do caso}

Paciente de 24 anos, sexo feminino, técnica em enfermagem, não ativa, com histórico de depressão, tinha pensado em "retirar" a própria vida no passado. Foi encontrada em sua casa, caída ao chão, chorosa, referindo e apontando ter injetado no músculo gramocil $\circledast$ (Paraquat), do seu lado foram encontrado uma seringa de $5 \mathrm{ml}$ com uma agulha acoplada e ain- da com restos do produto, e também foi encontrado próximo a vítima um do litro do veneno, foi levada imediatamente ao pronto socorro na cidade onde residia no dia 12 de março de 2013, ficando em observação por 24 horas, os exames revelaram, hemoglobina: $12,1 \mathrm{mg} / \mathrm{dl}$; hematócrito: $37,4 \%$; leucócitos: $17.600 \mathrm{~mm} / 3$; plaquetas 140.000; uréia: $18 \mathrm{mg} / \mathrm{dL}$; creatinina: $1,2 \mathrm{mg} / \mathrm{dL}$; aminotransferase de aspartate (AST): 25U/L; aminotransferase de alanine (ALT): 30U/L; Creatinofosfoquinase $(\mathrm{CPK}): 168 \mathrm{UI} / \mathrm{ml}$; potássio sérico $(\mathrm{K}+)$ : $3.0 \mathrm{mE} / q$ e sódio sérico $(\mathrm{Na}+) 143 \mathrm{mE} / \mathrm{q}$.

No segundo dia, foi encaminhada há um Pronto Socorro (PS) de um município que é referência em saúde, chegando de ambulância, adentrando a unidade deambulando, com Glasgow de 15/15, relatando ter injetado veneno Gramocil ${ }^{\circledR}$ no músculo e com expressão facial de preocupação ou seja arrependimento, pressão arterial(PA) de 120/80mmHg, frequência cardíaca(FC) de 80bpm, frequência respiratória(FR) de 14irpm, saturação de oxigênio(SatO2)96\%, AST: 142U/L, ALT:77U/L, uréia: $68 \mathrm{mg} / \mathrm{dL}$, creatinina: $3,1 \mathrm{mg} / \mathrm{dL}$, revelando assim de acordo com os resultados laboratoriais comprometimento da função hepática e renal, porém mantendo ainda estabilidade hemodinâmica, além da evolução progressiva com a metabolização do $\mathrm{PQ}$ pelo organismo da vítima, ao final do segundo dia, novos exames revelaram diminuição de hemoglobina para $10.9 \mathrm{mg} / \mathrm{dL}$ e hematócrito para $32.4 \%$, vale ressaltar que a paciente teve hormônio gonadotrofina coriônica (beta-HCG) negativo.

No terceiro dia, a vítima foi encaminhada a UTI mantendo nível de consciência, cefaleia de forte intensidade, expressão facial "triste", taquidispneia (32irpm), oligúria, hipocorada, hiperemia e induração na região do bíceps esquerdo com início de área necrosante, relatando ser naquela região onde injetou o $\mathrm{PQ}$ por via parenteral, foi passado cateter venoso central duplo lúmen e administrado drogas como metilpredinisolona125mg endovenoso 6/6h, N-acetilcisteína(Flui- 
Tabela-1 Parâmetros hemogasométricos de um paciente intoxicado por Paraquat parenteral atendido por um hospital público no interior de Rondônia/Brasil. 2018.

\begin{tabular}{lccccc}
\multicolumn{5}{c}{ Dias da semana } \\
Variáveis & $14 / 03$ & $15 / 03$ & $16 / 03$ & $17 / 03$ & $18 / 03$ \\
\hline $\mathrm{pH}$ & 7.38 & 7.31 & 7.25 & 7.33 & 7.19 \\
\hline $\mathrm{PaO2}$ & 55.2 & 62.2 & 53.9 & 61.6 & 52.9 \\
\hline $\mathrm{PaCO2}$ & 28.1 & 36.8 & 39.0 & $\mathrm{X}^{*}$ & $\mathrm{X}^{*}$ \\
\hline $\mathrm{HCO} 3$ & 15.2 & 18.3 & 16.7 & 25.7 & 39.0 \\
\hline $\mathrm{BE}$ & -8.5 & -7.1 & -9.8 & $-0,3$ & 8.8 \\
\hline SatO2 & 87.4 & 88.5 & 80.5 & 84.6 & 68.6
\end{tabular}

Fonte: os autores, 2020

Legenda: $X^{*}$ dados não encontrados no prontuário.

Tabela-2 Análise das enzimas renais de um paciente intoxicado por Paraquat parenteral atendido por um hospital público no interior de Rondônia/Brasil. 2018.

$\begin{array}{lccccc}\text { parenteral atendido por um hospital público no interior de Rondônia/Brasil. } 2018 . \\ \text { Variáveis } & 14 / 03 & 15 / 03 & 16 / 03 & 17 / 03 & 18 / 03 \\ \text { Ureia } & 87 & 89 & 140 & 170 & 214 \\ \text { Creatinina } & 5.7 & 6.7 & 8.3 & 9.0 & 10.3\end{array}$

Fonte: os autores, 2020

Tabela 3 Distribuição dos diagnósticos e intervenções de enfermagem de acordo com a CIPE $\Theta(13)$ a vítima de intoxicação parenteral por PQ. 2018

\section{Diagnósticos de enfer- magem}

$\begin{array}{ll} & \text { Atentar para o risco de suicídio. } \\ & \text { Orientar paciente sobre as atividades da equipe multidisciplinar. } \\ \text { Depressão } & \text { Promover segurança e conforto. } \\ & \text { Reduzir estímulos ambientais. } \\ \text { Solicitar acompanhamento psicológico. } & \text { Usar uma abordagem calma e segura. }\end{array}$

Angústia

$\begin{array}{ll} & \text { Avaliar nível de ansiedade. } \\ & \text { Avaliar o conhecimento e as expectativas do paciente. } \\ & \text { Encorajar a paciente a expressar suas inquietações. } \\ \text { Ensinar atividades que diminuam a ansiedade. } \\ \text { Estabelecer relação de confiança com o paciente. } \\ \text { Estimular a comunicação com o paciente. } \\ \text { Usar uma abordagem calma e segura. } \\ \text { Investigar o nível de ansiedade do paciente. } \\ \text { Manter ambiente seguro. } \\ \text { Orientar terapia de orientação para a realidade. } \\ \text { Oportunizar a família para expressar os seus sentimentos e pre- } \\ \text { Ocupações. } \\ \text { Ouvir a família. }\end{array}$

micil) 01 ampola endovenosa, ácido ascórbico 03 ampolas no soro.

No quarto dia, com piora do estado geral, por apresentar FR de 50 irpm, confusão mental, palidez cutânea geral e acidose metabólica importante, foi realizado intubação orotraqueal (IOT) instalado sedoanalgesia em dripping (dormindo $20 \mathrm{ml} / 05 \mathrm{mg} / \mathrm{ml}$ e fentanil $20 \mathrm{ml} / 50 \mathrm{mcg} /$ $\mathrm{ml})$. Foi indicado pelo médico intensivista hemodiálise de urgência, porém a empresa que prestava serviço de hemodiálise não tinha disponível tecnologia beira leito na época da admissão da paciente na UTI, vale ressaltar que essa empresa era conveniada com a Secretaria de Saúde de Rondônia(SESAU) através do convênio via SUS, que tem sua estrutura física externa a UTI. Diante da complexidade e instabilidade hemodinâmica a paciente não teve condições de ser removida da UTI para fazer a sessão de hemodiálise.

No quinto dia a paciente evoluiu para complicações sistêmicas corroborando com falência múltiplas de órgão (FMO), como acidose metabólica, midríase bilateral, instabilidade hemodinâmica como taquicardia e hipotensão severa sem resposta a fluidos e vasopressor, função respiratória com presença de taquidispneia roncos e estertores pulmonares difusos e presença de sangramento oral e via tubo orotraquela(TOT), hipoxemia de moderada a grave conforme revela os dados da gasometria arterial na tabela 1 , sistema renal com presença de colúria, oligoanúria em anasarca, e falência renal grave conforme revelou as enzimas renais na tabela 2. A paciente teve desfecho clínico desfavorável a todas as intervenções implementadas pela equipe multiprofissional, principalmente as manobras de RCPC, evoluiu a óbito 5 dias após a intoxicação parenteral.

\section{DISCUSSÃO}

Entre as diversas doenças que acometem a população no mundo a depressão é responsável por 4,3\%, e está entre as maiores e principais causas de incapaci- 
Auscultar sons respiratórios, observando presença de ruídos adventícios. Aspirar às vias aéreas mediante ausculta pulmonar.

Dispneia

Avaliar a perfusão tissular.

Manter a cabeceira da cama elevada.

Avaliar os sinais de cianose periférica e central.

Posicionar paciente no leito de forma confortável.

Hipóxia por congestão Coletar e avaliar a gasometria arterial. Implementar ações para minimizar a hipoxemia

Monitorar o padrão respiratório.

Monitorar os sinais vitais.

Observar o nível de consciência.

Observar perfusão periférica.

Respiração prejudicada Verificar a saturação do oxigênio pela oximetria de pulso ou gasometria.

Elevar a cabeceira da cama.

Auscultar sons respiratórios, observando presença de ruídos adventícios.

\begin{tabular}{|c|c|}
\hline Troca de gases prejudicada & $\begin{array}{l}\text { Aspirar às vias aéreas, quando necessário. } \\
\text { Avaliar a frequência e a profundidade respiratória de forma con- } \\
\text { tínua. } \\
\text { Manter elevação da cabeceira do leito a } 35-45^{\circ} \text {. } \\
\text { Monitorar gasometria arterial e os sinais de desequilíbrio acido- } \\
\text { básico. } \\
\text { Monitorar o batimento de asas do nariz, as retrações torácicas } \\
\text { e a cianose. } \\
\text { Monitorar sinais de congestão pulmonar e sistêmica. }\end{array}$ \\
\hline Desequilíbrio de eletrólitos & $\begin{array}{l}\text { Monitorar resultados laboratoriais (eletrólitos). } \\
\text { Monitorar pressão arterial e frequência cardíaca. } \\
\text { Controlar sinais de hidratação. } \\
\text { Controlar, rigorosamente, a terapia com líquidos e eletrólitos. }\end{array}$ \\
\hline Edema & $\begin{array}{l}\text { Avaliar a congestão pulmonar pela ausculta. } \\
\text { Avaliar as condições da pele e a perfusão. } \\
\text { Mudar o decúbito a cada duas horas. } \\
\text { Examinar condições de pulsos periféricos. }\end{array}$ \\
\hline
\end{tabular}

Risco de desequilíbrio de eletrólitos

Monitorar o débito urinário, descrevendo a diurese quanto ao volume, coloração e frequência.

Monitorar resultados dos exames laboratoriais (eletrólitos).

Condição nutricional prejudicada

Monitorar a ingestão da dieta.

Monitorar os sinais vitais.

Estimular a eliminação urinária.

Eliminação urinária reduzida

Monitorar a eliminação urinária, incluindo frequência, quantidade, cor e odor.

Registrar ingestão e eliminação de líquidos.

Orientar respirações profundas quando apresentar náuseas.

Náusea

Promover higiene oral frequentemente.

Proporcionar alimentação satisfatória.

Repor fluidos orais com líquidos frios.

Avaliar as características do vômito quanto à volume, coloração e odor.

Vômito
Avaliar o estado de hidratação do paciente.

Higienizar a cavidade oral após o vômito.

Posicionar a cabeça do paciente lateralizada.

Posicionar o paciente em semi-fowler para prevenir aspiração dades em mulheres. E associado a quadros depressivos vem o suicídio como um fenômeno global, sendo a principal causa de morte entre adolescentes de 15 a 19 anos.(8) A depressão e o suicídio implicam na influência de fatores biopsicossociais, genéticos, culturais e ambientais, sendo importante indicador da qualidade de vida da sociedade geral. ${ }^{(15)}$

A intoxicação exógena é um problema universal de saúde pública, acredita-se que 1,5 a $3 \%$ da população intoxicam-se todos os anos e os agrotóxicos estão entre os vilões na tentativa de suicídio principalmente pela facilidade de acesso que a população tem a esses produtos. No Brasil, isto representa aproximadamente 4.800 .000 casos novos a cada ano, destes, 0,1 a 0,4\% das intoxicações resultam em óbito. ${ }^{(15)}$

As vias de exposição a essas substâncias, mais utilizadas pelo ser humano, são a via gastrintestinal, respiratória e cutânea. Sendo que, as vias pulmonares e cutâneas são mais importantes na toxicologia ocupacional e ambiental, e a gastrintestinal em toxicologia de alimentos e de medicamentos, e nas intoxicações intencionais suicídios e homicídios. ${ }^{(16)}$

Geralmente em humanos, a ingestão de uma quantidade significativa de $P Q$, leva a morte dentro de duas a três semanas, como resultado de insuficiência renal aguda, hepática e, principalmente, insuficiência respiratória causada por inflamação pulmonar e fibrose. ${ }^{(6,17)}$

O PQ apresenta elevada toxicidade, depois de absorvido e distribuído pelos tecidos. ${ }^{(17)}$ Com isso, resulta a sua elevada capacidade de reagir com o oxigênio que, através de reações de oxidação-redução, leva à produção de radicais livres altamente lesivos para as células. A maioria dos autores sugere que a toxicidade do PQ resulta da sua enorme capacidade de reagir com o oxigênio. ${ }^{(18)}$

A clínica de um paciente vítima de intoxicação parenteral por PQ difere da intoxicação oral, pois pacientes que ingerem $\mathrm{PQ}$ sofrem de úlceras orais, hemoptise e sintomas gastrointestinais, como 


Integridade da pele preju-
dicada

Avaliar a evolução de lesões.

Avaliar a hidratação da pele.

Avaliar a necessidade de curativo e cobertura para a lesão.

Avaliar a região afetada, quanto aspecto, coloração, tecido cicatricial, secreção, odor e tipo de curativo.

Proporcionar posicionamento adequado.

Necrose

Realizar curativo diário.

Realizar desbridamento mecânico do material desvitalizado.

Supervisionar a pele circunvizinha à lesão.

Avaliar cor, temperatura e textura da pele.

Avaliar nível de consciência.

Risco de choque

Coletar lactato sérico.

Monitorar débito urinário.

Monitorar pressão venosa central (PVC).

Realizar rodízio de punção das falanges distal para a verificação

Risco de glicemia instável

da glicemia capilar.

Monitorar sinais de hipoglicemia.

Monitorar sinais de hiperglicemia.

Avaliar resposta a termorregulação.

Monitorar diminuição do nível de consciência.

Hipertermia

Monitorar e registrar sinais neurológicos.

Monitorar nível de consciência.

Avaliar a função cognitiva.

Avaliar o nível de consciência a cada duas horas.

Avaliar os reflexos.

Consciência prejudicada

Avaliar os sinais vitais.

Monitorar débito e eliminação urinária.

Avaliar o padrão das pupilas com a aplicação do pupilômetro.

Monitorar as alterações no nível de consciência, por meio da escala de coma de Glasgow.

Fonte: Nóbrega (2018). ${ }^{(13)}$

náuseas, vômitos, diarreia e sangramento gastrointestinal. ${ }^{(6)}$

A intoxicação por via parenteral é rara, porém, letal. ${ }^{(17)}$ Segundo pesquisadores ${ }^{(18)}$, quando a vítima decide por esta via, está sempre associado a tentativa de suicídio. Conforme relatado, a vítima já havia demostrado sinais de alerta como o de tentar "ceifar" a própria vida em outros momentos. Segundo Ministério da Saúde ${ }^{(10)}$, pensamentos e sentimentos de querer interromper a própria vida podem ser insuportáveis e difícil saber o que fazer e como superar esses sentimentos e a melhor recomendação é a prevenção e procurar ajuda. A $\mathrm{OMS}^{(8)}$ recomenda, que uma das estrategias é melhorar a gestão clínica e os cuidados de saúde mental de pessoas intoxicadas por agrotóxicos em estabelecimentos de saúde em diferentes níveis.
Existem poucos dados clínicos sobre a exposição parenteral por $\mathrm{PQ}$. O raro caso ora proposto mostrou evolução e progressão clínica contínua e de forma gradativa conforme foi ocorrendo a distribuição e metabolização do PQ pelo organismo.

A concentração do PQ em cada tecido relaciona-se com a vascularização deste a concentração do herbicida no plasma, sendo mais elevadas nos pulmões e nos rins. Contudo, o principal dano ocorre a nível pulmonar, culminando em falência respiratória, hipoxemia e morte. ${ }^{(6)}$

Em consequência das lesões no fígado, há redução do volume plasmático, pela diurese ou pela deslocação de fluidos para o lúmen intestinal, e com isso pode verificar hiperazotemia, hiponatremia, hipocalemia, proteinúria, hematúria, piúria, oligúria/anúria, glicosúria. ${ }^{(19,20)}$
Isso vai de encontro as manifestações e alterações laboratoriais que a vítima apresentou como zotemia, hipocalemia, hematúria e oligoanúria.

A lesão renal prejudica a principal via de excreção de $P Q$, com isso possibilita um contato mais prolongado do organismo com elevadas concentrações desse composto, o que contribui de forma significativa para a mortalidade. ${ }^{(19)}$

$\mathrm{O}$ PQ exerce maior toxicidade nos pulmões, haja visto as suas altas concentrações celulares, serem superiores à concentração no plasma. A toxicidade do PQ a nível pulmonar dá-se em duas fases distintas, a fase aguda, que causa dano não só nos pulmões, mas também no fígado, rins e coração, podendo o doente morrer devido a falência multiorgânica. Os doentes que resistem a esta primeira fase ficam sujeitos a uma fase tardia caracterizada quase exclusivamente por dano pulmonar, em que se desenvolve fibrose pulmonar extensa. ${ }^{(20)}$

A fibrose pulmonar evidencia-se, clinicamente, por um quadro de falência respiratória com tosse, taquipneia, dispnéia progressiva, hipoxemia e cianose periférica, perfusão tissular periférica $>5 \mathrm{seg}$, que pode culminar em morte. A ventilação mecânica invasiva (VMI) e não invasiva artificial (VMNI), ou outros métodos de oxigenioterapia seja de baixo ou alto fluxo, não são capazes de reverter a hipoxemia e atrasar o desfecho clínico que culmina com $100 \%$ de mortalidade. ${ }^{(20)}$

Segundo pesquisadores ${ }^{(20)}$, deve-se evitar ao máximo a oferta de oxigênio, o que favorece a formação de radicais livres, agravando a toxicidade induzida pelo PQ. Entretanto outro estudo(19), revela que em casos de insuficiência respiratória grave, deve ser usado a VMI para minimizar o sofrimento e aliviar a fadiga do paciente.

Paciente com intoxicação parenteral por $\mathrm{PQ}$, não tem irritação direta da mucosa, porém apresentam sintomas, como náuseas e vômitos, que pode ser explicado pelo efeito sistêmico do paraquat no sistema nervoso central. Os mais comuns são os sintomas locais de pele ou vasos. 
(17) O caso supracitado a paciente, desenvolveu no local da aplicação, induração na região do bíceps esquerdo face interna com início de área de necrose de coagulação e precisou de cuidados especiais com a pele pela equipe de enfermagem em relação a cobertura e desbridamento da área necrosada.

As lesões na pele pode ser explicado pela reação local devido à ocorrência de extravasamento trivial da solução de PQ para o tecido macio adjacente e / ou lesão local do vaso sanguíneo como a flebite, relacionada especificamente com a injeção. Os efeitos toxicológicos sistêmicos também são suspeitos de ter início mais rápido em pacientes com intoxicação intravenoso do que em pacientes com ingestão oral. ${ }^{(17)}$

A equipe de enfermagem tem papel fundamental no cuidado ao paciente vítima de intoxicação. Esse cuidado deve ser baseado em princípios e diretrizes que norteiam ações sistematizadas e direcionadas, buscando medidas para prevenir e evitar complicações de forma precoce decorrentes da substância envolvida. ${ }^{(21)}$ Para isso se faz necessário a utilização do processo de enfermagem que é composto por 5 etapas e que o estudo ora proposto buscou trazer somente um direcionamento aos profissionais da saúde voltado a primeira a e segunda etapa do processo de enfermagem.

A atuação do enfermeiro em conjunto com a equipe multiprofissional, em todas as etapas de assistência, seja preventiva, curativa emergencial ou de acompanhamento durante a internação e após a alta hospitalar, repercute favoravelmente para a redução dos índices de recidiva destas intoxicações.

A paciente não teve condições clínicas e hemodinâmicas de fazer hemodiálise, tendo em vista a clínica de hemodiálise ser externamente a UTI, sendo contraindicado transporta-la por instabilidade e no período de internação não havia o serviço de hemodiálise beira leito implantado dentro da UTI.

Segundo Schmitt et al., o PQ não é um tóxico adequadamente dialisável, uma vez que tem um elevado peso molecular e não se encontra ligado às proteínas. ${ }^{(22)}$ Em doentes com insuficiência renal aguda

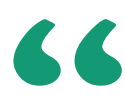

O tratamento proposto na unidade de terapia intensiva, foi a N-Acetilcisteína que é um reagente sulfidrílico, com potenciais efeitos benéficos na intoxicação por PQ através de diversos mecanismos, eliminando espécies reativas de oxigênio, aumentando a glutationa e reduzindo a inflamação, peroxidação lipídica e apoptose. ${ }^{(24)}$

O uso de glucocorticóides e ciclofosfamida combinados, vem desempenhando um papel importante no aumento da sobrevivência nas intoxicações por PQ. O esquema terapêutico mais amplamente estudado inclui ciclofosfamida, metilprednisolona e dexametasona, ${ }^{(23)}$ agregando na ação anti-inflamatória dos glucocorticóides e na ação imunossupressora da ciclofosfamida.

Indivíduos intoxicados por PQ apresentam uma taxa de mortalidade compreendida entre $63 \%$ e $70 \%$ e um curto período de sobrevivência, dependendo da dose ingerida e absorvida, das complicações consequentes da intoxicação, e da escolha de tratamento eficaz. ${ }^{(6,25)}$

Segundo outros pesquisadores, ${ }^{(26)} \mathrm{O}$ $P Q$, quando administrado pela via parenteral a mortalidade é de $100 \%$.

\section{CONCLUSÃO}

\section{sanguíneo como a flebite, relacionada especificamente com a injeção.}

A pesquisa mostrou o quão importante é a identificação dos sinais de alertas relacionado a comportamentos suicidas, assim é preciso desmistificar a problemática e apontar para a importância de olhar atento e contínuo para os profissionais de saúde e população em geral. E nesse sentido, através do caso clínico, descreveu-se a evolução clínica da paciente, que apresentou comportamento e sinais de alerta ao suicídio, comprometimento hepatorrenal e manifestações clínicas respiratórias severas, ocasionando a faIha multiorgânica, culminando com alta mortalidade.

A intoxicação parenteral por $P Q$, é sintomática, deve-se considerar a hemodiálise. No entanto, esses pacientes têm um prognóstico ruim em termos de lesão pulmonar, por isso é improvável que a técnica altere o desfecho. ${ }^{(23)}$ um problema com grande incidência e alta letalidade principalmente na cidade de cerne desta pesquisa, sendo assim é 
de suma importância pesquisas e estudos na área, com intuito de ampliar o conhecimento sobre o tema, auxiliando a equipe de saúde a encontrar maneiras de implementar cuidados que evite a evolução rápida do $\mathrm{PQ}$ e assim possam melhorar a assistência ao paciente grave.

Foram implementados os principais diagnósticos de enfermagem com base na nomenclatura da CIPE $®$, assim diante da escassez de estudos envolvendo vítimas de intoxicação parenteral por PQ e que sugere novos estudos explorando mais as ações de enfermagem com foco na sistematização da assistência de enfermagem.
A equipe multidisciplinar é responsável pelos pacientes na UTI, entretanto, o enfermeiro é o único profissional junto com sua equipe que está 24 horas beira do leito 7 dias da semana, assim, todos devem buscar práticas baseada em evidências acerca das características predominantes e evolução do paciente intoxicado por $\mathrm{PQ}$, estando preparado para implementar as intervenções necessárias, que minimizem a evolução do $\mathrm{PQ}$, pelo organismo da vítima.

Foi evidenciado no Brasil uma escassez de pesquisas atuais abordando a intoxicação por $\mathrm{PQ}$, assim o resultado des- te relato pode despertar a comunidade científica a buscar novas pesquisas, que podem contribuir com ações no contexto preventivo, além de melhorar as práticas baseado em evidencias. Sugere também que as instituições formadoras na área da saúde, não só discutem, mas, que introduzam em suas grades curriculares conteúdos como depressão, suicídio e intoxicações exógenas.

\section{CONFLITO DE INTERESSE}

Os autores declaram não haver nenhum conflito de interesse na atual pesquisa.

\section{Referências}

1. Rodrigues, DS; Rebouças DS; Teles AMS; Conceição Filho JN; Guimarães CRR; Santana OAM et al., Apostila de Toxicologia Básica. Centro de referência estadual em toxicologia. Centro de Informações Antiveneno da Bahia - CIAVE. Salvador, 2009

2. Peres F, Rozemberg B, Alves SR, Moreira JC, Oliveira Silva JJ. Comunicação relacionada ao uso de agrotóxicos em região agrícola do Estado do Rio de Janeiro. Rev Saúde Pública. 2001; 35:564-70

3. Melchiorri, D. Reiter RJ; Sewerynek E; Hara M; Chen L; Nisticò G. Paraquat toxicity and oxidative damage. Biochemical Pharmacology, v.51, p.1095-1099, 1996.

4. Crugeiras, P; Malho, A; Nunez, D; Sousa, A; Neves, P; Patraquim, R. Sucesso terapêutico na intoxicação por paraquat. Rev Port Med Int. Vol 16(2), 2009. 5. Naito, $\mathrm{H}$, Yamashita, M. Epidemiology of paraquat in Japan and new safe formulation of paraquat. Human Toxicology, v.6, n.1, p.87-88, 1987.

6. Dinis-Oliveira, RJ Duarte, Sánchez-Navarro JÁ Remião, AF, Bastos ML, Carvalho F. Paraquat poisonings: Mechanisms of lung toxicity, clinical features, and treatment. Crit Rev Toxicol. 38(1):13-71, 2008.

7. Pinheiro I. Intoxicação por Paraquat - Revisão bibliográfica. Hospital Santa Maria, Lisboa. 2002

8. Organização Mundial de Saúde (OMS). Preventing suicide: a global imperative. Genebra. WHO. 2014.

9. Brasil. Ministério da Saúde. Sistema Nacional de Informações Tóxico-Farmacológicas. Casos registrados de intoxicação humana por agente tóxico e circunstância. [Acesso em 13 de junho de 2020] http://www.cict.fiocruz.br/ intoxicacoeshumanas/2001/brasil2001

10. Brasil. Ministério da Saúde. Boletim Epidemiológico: Suicídio: tentativas e óbitos por intoxicação exógena no Brasil, 2007 a 2016. Secretaria de Vigilância em Saúde. Ministério da Saúde. Volume 50 | № 15 | Jul. 2019.

11. Silva Darlan dos Santos Damásio, Tavares Natália Vieira da Silva, Alexandre Alícia Regina Gomes, Freitas Daniel Antunes, Brêda Mércia Zeviani, Albuquerque Maria Cícera dos Santos de et al . Depressão e risco de suicídio entre profissionais de Enfermagem: revisão integrativa. Rev. esc. enferm. USP [Internet]. 2015 Dec [cited 2020 Jan 17] ; 49( 6 ): 1023-1031. Available from: http://www.scielo.br/scielo.php?script=sci_arttext\&pi$\mathrm{d}=$ S0080-62342015000601023\&lng=en. http://dx.doi.org/10.1590/S0080623420150000600020 .

12. Horta, W.A. Processo de Enfermagem. São Paulo: EPU, 2011. Internacional Council of Nurses. International Classifi-cation for Nursing practice Version 2. Geneva, Switzerland: Inter-national Council of Nurses. 2009.

13. Nóbrega MML, Moreira AM, Cunha ACR, (org). Nomenclatura de diagnósticos, resultados e intervenções de enfermagem: para pacientes hospita- lizados em unidades clínicas, utilizando a CIPE®. João Pessoa: Ideia, 2018. 14. Instituto Brasileiro de Geografia e Estatística (IBGE).2018. Acesso em 13 de abril 2020. Disponível em: https://cidades.ibge.gov.br/brasil/ro/cacoal/ panorama.

15. Heck RM, Kantorski LP, Borges AM, Lopes CV, Santos MC, Pinho LB. Ação dos profissionais de um centro de atenção psicossocial diante de usuários com tentativa e risco de suicídio. Texto Contexto Enferm [Internet]. 2012 [citado 2020 jan. 17]; 21(1):26-33.

16. Zambolim CM, Oliveira TP, Hoffmann NA, Vilela CEB, Neves D, Anjos FR, et al. Perfil das intoxicações exógenas em um hospital universitário. Rev Med Minas Gerais. 2008;18(1):5-10. [acesso em 20 de fevereiro de 2020].

17. Thorne PS. Occupational toxicology. In: Klaassen CD. Casarett and Doull'stoxicology: the basic science of poisons. 8th ed. New York:McGraw-Hill Education, 2013. 1454 p. Chapter 34.

18. Choi Y , Cho K, Yoon S, Lee H. A case of paraquat intoxication caused by intravenous injection. The American Journal of Emergency Medicine , Volume 26 , Issue $7,836 . e 3$ - 836.e4. 2008.[acesso em 25 de janeiro de 2019] DOI: http://dx.doi.org/10.1016/j.ajem.2007.12.016

19. Chen HW, Tseng TK, Ding LW . Intravenous paraquat poisoning. J Chin Med Assoc. 2009 Oct; 72 (10): 547-50. DOI:10.1016 / S1726-4901 (09) 70426-5.

20. Castro R, Prata C, Oliveira L, Carvalho MJ, Santos J, Carvalho F, et al. Paraquat intoxication and hemo carbo perfusion. Acta Med Port 2005;18(6):423-31

21. SANTOS RR, Almeida Neto OP, Cunha CM. Perfil de vítimas de intoxicações exógenas agudas e assistência de enfermagem. Rev Enferm Atenção Saúde [Online]. Ago/Dez 2015; 4(2):45-55

22. Schmitt GC, Paniz C, Grotto D, Valentini J, Schott KL, Pomblum VJ, et al. Aspectos gerais e diagnóstico clinico laboratorial da intoxicação por paraquat. J Bras Patol e Med Lab. 2006;42(4):235-43.

23. Monteiro R, Correia L, Simão A, Carvalho A, Nascimento-Costa J. Intoxicação por paraquat: artigo de revisão. Rev da Soc Port Med Interna. 2011;18(3):173-80.

24. Gawarammana IB, Buckley NA. Medical management of paraquat ingestion. Br J Clin Pharmacol. 2011;72(5):745-57.

25. Dinis-Oliveira RJ, Sarmento A, Reis P, Amaro A, Remião F, Bastos ML, et al. Acute paraquat poisoning: report of a survival case following intake of a potential lethal dose. Pediatr Emerg Care. 2006;22(7):537-40.

26. Souza LP, Lima MG, Neves DS, Romanholo HSB, Viana TCT, Romanholo RA, Almeida FM. Poisoning by parental PQ: case report in a city of Amazonia/ Brazil. Review of Research. Volume 4 | Issue 6 | March 2015 | Online \& Print. 